\title{
VCSEL based on InAs Quantum-Dashes with a lasing operation over a 117-nm wavelength span
}

\author{
F. Taleb, C. Levallois, C. Paranthoën, J.-P. Gauthier, N. Chevalier, M. Perrin, \\ Y. Léger, O. De Sagazan and A. Le Corre
}

\begin{abstract}
We report an InP based vertical cavity surface emitting lasers (VCSEL) achieving lasing operation between 1529 $\mathrm{nm}$ and $1646 \mathrm{~nm}$. This optically-pumped VCSEL includes a widegain bandwidth active region based on InAs quantum dashes and wideband dielectric Bragg mirrors. Based on a wedge microcavity design, we obtain a spatial dependence of the resonant wavelength along the wafer, enabling thus to monitor the gain material bandwidth. We demonstrate a $117 \mathrm{~nm}$ continuous wavelength variation of the VCSEL emission, a consequence of the important and wide gain afforded by the use of optimized quantum dashes.
\end{abstract}

Index Terms- Quantum dash lasers, Semiconductor lasers, Vertical cavity surface emitting lasers.

\section{INTRODUCTION}

$\mathrm{W}$ AVELENGTH-TUNABLE vertical-cavity surface-emitting laser (VCSEL) is a promising light source for use in fiber Bragg-grating sensors [1], gas spectroscopy [2], telecommunications [3] and optical coherence tomography systems [4]. For these applications, single-mode laser emission with continuous and a wide wavelength tuning are required. VCSEL is an attractive laser source due to its short cavity design and its inherent longitudinal single-mode behavior to provide a continuous wavelength tuning. However, to achieve a wavelength tuning range higher than $50 \mathrm{~nm}$, wideband Distributed Bragg Reflectors (DBR) and active materials with a large gain bandwidth are also required. Quantum wells (QWs) are predominantly used as a wide-gain material in tunable VCSEL active regions. Recently, continuous wavelength tuning over $100 \mathrm{~nm}$ has been demonstrated on electrically pumped or optically pumped VCSELs including QWs and a microelectromechanical-system [4][5]. However, quantum dots (QDs) or quantum dashes (QDHs) are also well suited to be used as wide-gain material in tunable VCSEL. Such nanostructures offer an important inhomogeneous broadening due to their size distribution and are very promising for tunable VCSEL, super luminescent diode (SLD)

Manuscript received June 26, 2013.

F. Taleb, C. Levallois, C. Paranthoën, N. Chevalier, M. Perrin, Y. Léger, and A. Le Corre are with INSA, FOTON, UMR-CNRS 6082, F-35708 Rennes, France and also with the Université Européenne de Bretagne, France (e-mail :christophe.levallois@insa-rennes.fr).

J.-P. Gauthier and O. De Sagazan are with GM-IETR UMR-CNRS 6164, Université de Rennes 1, F-35042 Rennes Cedex, France. or optical amplifiers with a wide-gain bandwidth. Using InPbased QDHs layers at a wavelength of $1.6 \mu \mathrm{m}$, a SLD with amplified spontaneous emission bandwidth as wide as $140 \mathrm{~nm}$ has already been reported [6]. More recently, optically pumped GaAs-VCSELs including QDs have achieved a $60 \mathrm{~nm}$ continuous wavelength variation at $1.25 \mu \mathrm{m}$ wavelength [7].

In this letter, we present an InP-VCSEL based on InAs QDH nanostructures operating at telecommunication wavelength. In addition to the VCSEL output polarization control related to the QDH we have already evidenced [8][9], we demonstrate that QDH inhomogeneously broadened gain enables a CW VCSEL emission from $1646 \mathrm{~nm}$ down to 1529 $\mathrm{nm}$, covering thus a spectral window as large as $117 \mathrm{~nm}$. This result is an experimental evidence of QDH large wavelength gain bandwidth suitable for widely tunable VCSEL applications.

\section{GROWTH AND PROCESSING}

VCSEL sample is grown by gas source molecular beam epitaxy on a 2 inches diameter $\operatorname{InP}(001)$ substrate. Active region consists of three groups of QDHs layers separated with InP spacers to be located at the position of the microcavity antinodes fields. Each group corresponds to six InAs QDH layers closely separated with $15 \mathrm{~nm} \mathrm{Ga}_{0.2} \mathrm{In}_{0.8} \mathrm{As}_{0.435} \mathrm{P}_{0.565}$ barriers. This quaternary alloy is also used to surround each group of QDHs layers, and acts as absorbing layers for the optical excitation of the device. Careful attention has been paid to design these layer thicknesses for each QDHs group to reach a homogeneous carrier injection within the structure. Details on QDH growth and morphology may be found in reference [8]. To go further, QDH growth has been carefully optimized in order to get a reproducible QDH emission wavelength layer to layer, compensating the natural wavelength shift related to the internal strain field mediation. As a consequence, we obtain a QDH integrated photoluminescence (PL) intensity being even slightly higher than conventional $1.55 \mu \mathrm{m}$ strained QWs. Details on this optimization will be the subject of another paper. Prior to the processing, PL measurements have been conducted on the VCSEL sample, at a low optical power density of $10 \mathrm{~W} / \mathrm{cm}^{2}$, and at a $1.064 \mu \mathrm{m}$ wavelength excitation. Such InP transparent wavelength enables to get PL properties of the three groups of six QDH layers by creating carriers in the GaInAsP barriers. As shown in Fig. 1, the QDHs wavelength emission is rather 


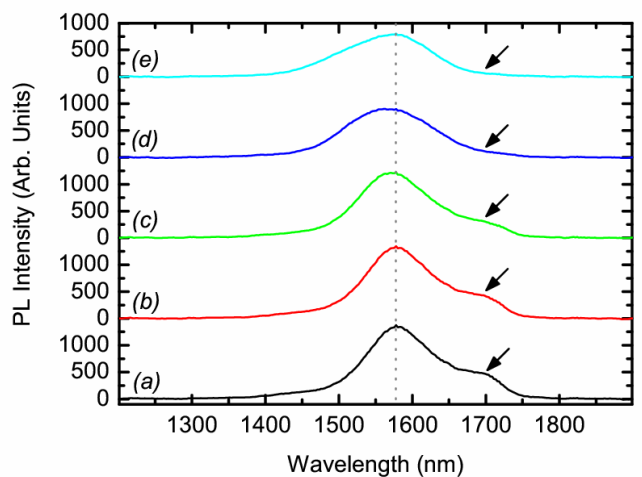

Fig. 1. PL spectra at $300 \mathrm{~K}$ under an incident power of $10 \mathrm{~W} / \mathrm{cm}^{2}$ and a $1.064 \mu \mathrm{m}$ laser wavelength measured at different positions from the center of the wafer to its edge. Spectra (a), (b), (c), (d) and (e) correspond to PL measurements at a distance of $5,7.5,10,12.5$ and $20 \mathrm{~mm}$ from the wafer center respectively. The vertical dotted line located at $1575 \mathrm{~nm}$ is only a guide for the eyes.

constant and centered at $1575 \mathrm{~nm}$ on the 2-inch InP substrate. Note that the arrow indicating a PL shoulder, is not ascribed to QDH but related to an underneath InGaAs stop-etch required in our VCSEL fabrication process. The PL linewidth slightly increases from 116 to $130 \mathrm{~nm}$, and the overall integrated intensity decreases only by a factor of 1.6 , from measurements done respectively from the center up to the edges of the wafer. Considering those PL measurements, we can assume that over the whole surface of the wafer, the QDH properties are nearly identical. This VCSEL active region based on such QDHs has been grown on a $1500 \mathrm{~nm}$ thick InP phase layer including a thickness gradient. This gradient has been created by a voluntary interruption of the sample rotation during the growth to achieve a cavity length decreasing from the center up to the edge of the wafer. This wedge cavity is expected to present a thickness variation estimated to be around $10 \%$, being appropriate to induce a significant variation of the resonant wavelength.

After the growth of the QDHs based active region, the VCSEL cavity is formed with wideband dielectric DBRs to allow a laser emission on a large spectral width. A first dielectric DBR constituted of six pairs of amorphous $\mathrm{Si}$ (a-Si) and amorphous $\mathrm{SiN}$ (a-SiN) is deposited by magnetron sputtering on the wafer. Considering the important optical index contrast of 1.85 and the optical losses of these layers, with optical simulations based on transfer matrix method we estimate the reflectivity to be greater than $99.5 \%$ at $1.55 \mu \mathrm{m}$, over a stopband of $250 \mathrm{~nm}$ for our bottom DBR including six periods. Thus, metallic layers of $\mathrm{Ti}$ and $\mathrm{Au}$ are deposited onto this first DBR and a $50 \mu \mathrm{m}$ thick copper film is electro-plated to form a metallic substrate. The whole InP substrate is then removed by mechanical and selective chemical etching. Finally, the InGaAs stop-etch layer is also removed by chemical etching, and a second dielectric DBR including four periods is deposited on the wedge cavity.

\section{QUANTUM-DASH VCSEL CHARACTERIZATION}

The Fig. 2 inset represents the schematic view of the QDH

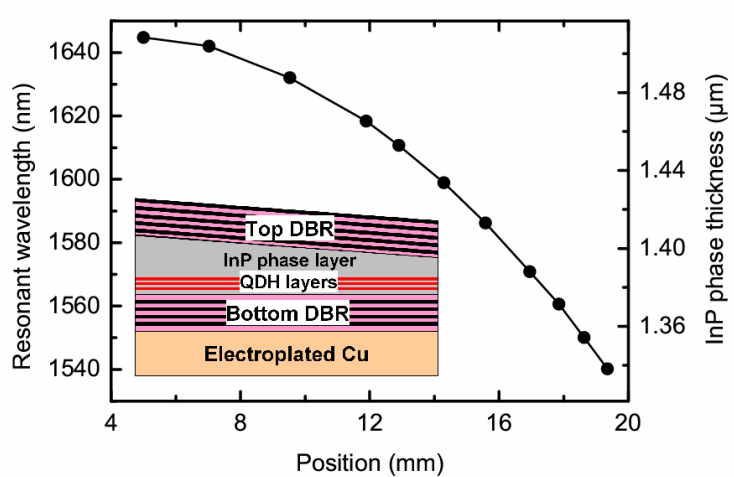

Fig. 2. Resonant wavelength of the VCSEL cavity as a function of position on the wafer. Positions indicated in $\mathrm{mm}$ correspond to the distance from the wafer center. Inset is a schematic cross-section of the fabricated QDH VCSEL.

VCSEL when fabrication process is completed. Prior to lasers characterizations, spontaneous emission from our device has been measured at room-temperature (RT) by pumping the QDH active region with a $980 \mathrm{~nm}$ continuous-wave (CW) laser diode. Those measurements have been done at low excitation power $(<5 \mathrm{~mW})$ on a $15 \mu \mathrm{m}$ diameter $\left(1 / \mathrm{e}^{2}\right.$ width) focused spot. Resonant wavelength and free spectral range as function of the position on the wafer have been analyzed to deduce the effective thickness gradient of the InP phase layer. A plot of the measured resonant wavelength and the deduced InP phase thickness as a function of the position on the wafer is shown in Fig. 2. The X-axis represents the radial position of the wafer from the center at $0 \mathrm{~mm}$ to the edge of the VCSEL sample at $20 \mathrm{~mm}$. Resonant wavelength continuously decreases from 1645 down to $1540 \mathrm{~nm}$, corresponding in a $165 \mathrm{~nm}$ decrease of the InP phase thickness, close to the $10 \%$ variation expected.

With the optical set-up described above, laser experiments in $\mathrm{CW}$ operation at RT have been performed at different positions on the VCSEL sample. Output spectra have been monitored with an optical spectrum analyzer by moving the

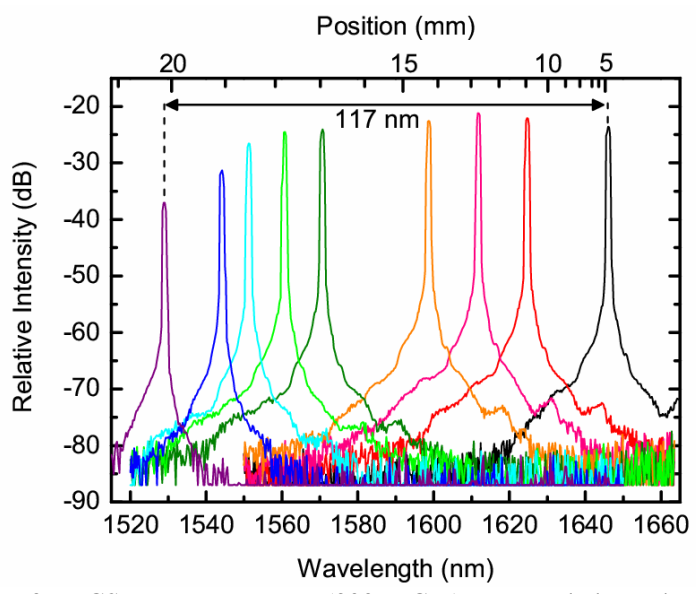

Fig. 3. VCSEL output spectra $(300 \mathrm{~K}, \mathrm{CW})$ measured above threshold at a constant pump power, for different position across the wafer (from 5 up to $20 \mathrm{~mm}$, from right to left respectively). The upper scale relies on the dependence of the position from the center of the wafer and the cavity resonant wavelength. The arrows illustrate the wide wavelength span of the VCSEL emission. 
pump spot along the radius of the wafer. The different lasing spectra, shown in Fig. 3, have been recorded above threshold at a $15 \mathrm{~mW}$ constant absorbed pump power (corresponding to a $8.5 \mathrm{~kW} / \mathrm{cm}^{2}$ pump power density). Close to the center of the wafer, the longest lasing wavelength measured is $1646 \mathrm{~nm}$. In agreement with spontaneous emission measurements, as the distance from the center increases, resonant wavelength is reduced, and lasing wavelength continuously decreases. Close to $16 \mathrm{~mm}$ from the wafer center, laser emission wavelengths are measured to be close to $1585 \mathrm{~nm}$. But because of numerous defects on the top DBR related to processing artifacts, VCSEL laser performances are poor, and data were not considered in the following. At the edge of the VCSEL sample, the shortest lasing wavelength measured is $1529 \mathrm{~nm}$, demonstrating a CW laser emission at RT in a very large spectral window of 117 $\mathrm{nm}$. Note that as previously demonstrated for such QDH based VCSEL, the output VCSEL intensity exhibits a stable and polarized laser emission over the whole spectral range, with a polarization ratio greater than $25 \mathrm{~dB}$ along the [1-10] crystallographic direction [8][9] (not shown here). Fig. 4 shows the output power versus incident pump power curves recorded for a few lasing wavelengths of the Fig. 3.

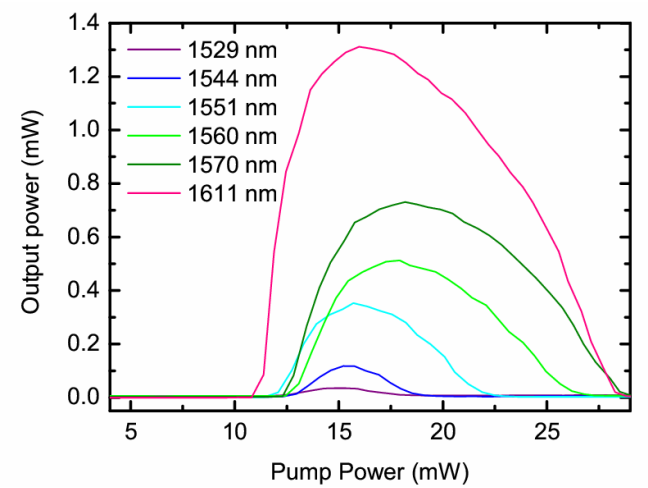

Fig. 4. CW VCSEL output power versus incident pump power for different positions across the wafer.

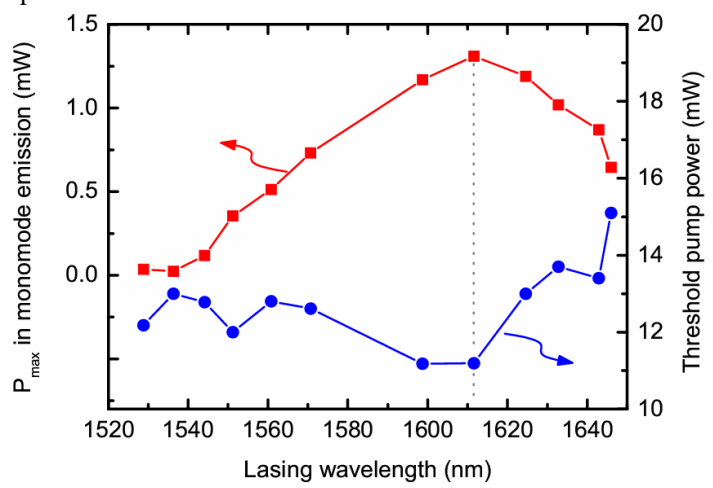

Fig. 5. Evolution of laser thresholds and maximum output powers (in monomode emission) as a function of emitted wavelength measured in $\mathrm{CW}$ operation.

As it can be seen, the laser slope efficiencies and thresholds depend on the emitted wavelength. The maximum achievable singlemode output power is mainly limited by thermal rollover and the pump spot diameter of $15 \mu \mathrm{m}$. For several emitted wavelengths, at a pump power greater than $17 \mathrm{~mW}$, higherorder transverse modes appear and VCSEL emission becomes multimode. The maximum achievable singlemode output powers and lasing thresholds for the different wavelengths are reported in Fig. 5. Minimum threshold and maximum output power have been obtained at a position of $13 \mathrm{~mm}$ away from the wafer center, corresponding to a lasing wavelength of 1611 $\mathrm{nm}$. Considering thus, the maximal achieved output power minus $3-\mathrm{dB}$ window, the threshold variation does not exceed $10 \%$, and the spectral window is covering the [1570-1640] nm range. Finally, from room temperature until the highest operation temperature of $50^{\circ} \mathrm{C}$, we estimate the characteristic temperature $\mathrm{T}_{0}$ to be $73 \mathrm{~K}$

\section{CONCLUSION}

A $1.6 \mu \mathrm{m} \mathrm{InP} \mathrm{VCSEL} \mathrm{continuous} \mathrm{wavelength} \mathrm{variation} \mathrm{of}$ $117 \mathrm{~nm}$ has been demonstrated, combining the use of a QDH active region, wideband dielectric DBRs and a wedge cavity. These experiment evidences the large and wide gain afforded by inhomogeneous QDH nanostructures. In association with a wavelength tuning mechanism, QDH VCSEL are expected to achieve very large tuning, exceeding $100 \mathrm{~nm}$, without any specific gain engineering of this active region.

\section{REFERENCES}

[1] C. F. R. Mateus and C. L. Barbosa, "Harsh environment temperature and strain sensor using tunable VCSEL and multiple fiber bragg gratings," in 2007 SBMO/IEEE MTT-S Int. Microwave and Optoelectronics Conf., pp. 496-498.

[2] B. Kogel, H. Halbritter, S. Jatta, M. Maute, G. Bohm, M.-C. Amann, M. Lackner, M. Schwarzott, F. Winter, and P. Meissner, "Simultaneous Spectroscopy of $\mathrm{NH}_{3}$ and $\mathrm{CO}$ Using a $50 \mathrm{~nm}$ Continuously Tunable MEMS-VCSEL," IEEE Sensors J, vol. 7, no. 11, pp. 1483-1489, Nov. 2007.

[3] A. Daly, C. Gierl, T. Gründl, C. Grasse, K. Zogal, D. Carey, P. Townsend, M.-C. Amann, P. Meissner, and B. Corbett, "10 Gbit/s transmission over $50 \mathrm{~km}$ of SMF using MEMS tunable VCSEL," Electron. Lett., vol. 48, no. 7, pp. 394-396, Mar. 2012.

[4] V. Jayaraman, G. D. Cole, M. Robertson, A. Uddin, and A. Cable, "High-sweep-rate $1310 \mathrm{~nm}$ MEMS-VCSEL with $150 \mathrm{~nm}$ continuous tuning range," Electron. Lett., vol. 48, no. 14, pp. 867-869, Jul. 2012 .

[5] C. Gierl, T. Gruendl, P. Debernardi, K. Zogal, C. Grasse, H. A. Davani, G. Böhm, S. Jatta, F. Küppers, P. Meissner, and M.-C. Amann, "Surface micromachined tunable $1.55 \mu \mathrm{m}$-VCSEL with $102 \mathrm{~nm}$ continuous single-mode tuning," Opt. Express, vol. 19, p. 17336, Aug. 2011 .

[6] B.S. Ooi, H.S. Djie, Yang Wang, Chee-Loon Tan, J. Hwang, XiaoMing Fang, J.M. Fastenau, A. Liu, G.T. Dang, and W.H. Chang, "Quantum Dashes on InP Substrate for Broadband Emitter Applications," IEEE J Sel. Topics Quantum Electron., vol. 14, no. 14, pp. 1230-1238, Jul./Aug. 2008.

[7] A. R. Albrecht, A. Stintz, F. T. Jaeckel, T. J. Rotter, P. Ahirwar, V. J. Patel, C. P. Hains, L. F. Lester, K. J. Malloy, and G. Balakrishnan, "1220 - 1280 nm Optically Pumped InAs Quantum Dot-Based Vertical External-Cavity Surface-Emitting Laser," IEEE J. Sel. Topics in Quantum Electron., vol. 17, no. 6, pp. 1787-1793, Nov./Dec. 2011.

[8] J. M. Lamy, C. Paranthoen, C. Levallois, A. Nakkar, H. Folliot, J. P. Gauthier, O. Dehaese, A. Le Corre, and S. Loualiche, "Polarization control of 1.6 $\mu \mathrm{m}$ VCSEL using InAs quantum dashes on $\operatorname{InP}(001), "$ Appl. Phys. Lett., vol. 95, p. 011117, 2009.

[9] J.-P. Gauthier, C. Paranthoen, C. Levallois, A. Shuaib, J.-M. Lamy, H. Folliot, M. Perrin, O. Dehaese, N. Chevalier, O. Durand, and A. Le Corre, "Enhancement of the polarization stability of a $1.55 \mu \mathrm{m}$ emitting vertical-cavity surface-emitting laser under modulation using quantum dashes," Opt. Express, vol. 20, no. 15, p. 16832, 2012. 